\title{
Global Stabilization of High-Order Time-Delay Nonlinear Systems under a Weaker Condition
}

\author{
Nengwei Zhang, ${ }^{1}$ Enbin Zhang, ${ }^{2}$ and Fangzheng Gao ${ }^{1}$ \\ ${ }^{1}$ School of Mathematics and Statistics, Anyang Normal University, Anyang 455000, China \\ ${ }^{2}$ Department of Information Engineering, Henan College of Finance and Taxation, Zhengzhou 450002, China \\ Correspondence should be addressed to Fangzheng Gao; gaofz@126.com
}

Received 25 May 2014; Accepted 28 July 2014; Published 28 August 2014

Academic Editor: Chengjian Zhang

Copyright (c) 2014 Nengwei Zhang et al. This is an open access article distributed under the Creative Commons Attribution License, which permits unrestricted use, distribution, and reproduction in any medium, provided the original work is properly cited.

Under the weaker condition on the system growth, this paper further investigates the problem of global stabilization by state feedback for a class of high-order nonlinear systems with time-varying delays. By skillfully using the homogeneous domination approach, a continuous state feedback controller is successfully designed, which preserves the equilibrium at the origin and guarantees the global asymptotic stability of the resulting closed-loop system. A simulation example is given to demonstrate the effectiveness of the proposed design procedure.

\section{Introduction}

Time-delay phenomena exist in many practical systems such as electrical networks, microwave oscillator, and hydraulic systems. It is well known that the existence of time delay often deteriorates the control performance of systems and even causes the instability of closed-loop systems [1]. Therefore, the control design and stability analysis of time-delay systems has been an active research area within the automation and control community. In recent years, by employing the Lyapunov-Krasovskii method or Lyapunov-Razumikhin method to deal with the time delay, control theory, and techniques for time-delay linear systems were greatly developed and many advanced methods have been made; see, for instance, [2-9] and reference therein. However, due to no unified method being applicable to nonlinear control design, many important and interesting control problems for timedelay nonlinear systems remain unsolved.

In this paper, we consider a class of high-order time-delay nonlinear systems described by

$$
\begin{aligned}
& \dot{x}_{i}(t) \\
& =x_{i+1}^{p_{i}}(t)+f_{i}\left(\bar{x}_{i}(t), x_{1}\left(t-d_{1}(t)\right), \ldots, x_{i}\left(t-d_{i}(t)\right)\right)
\end{aligned}
$$

$$
\begin{aligned}
& \dot{x}_{n}(t) \\
& =u^{p_{n}}(t)+f_{n}\left(\bar{x}_{n}(t), x_{1}\left(t-d_{1}(t)\right), \ldots, x_{n}\left(t-d_{n}(t)\right)\right),
\end{aligned}
$$

where $x(t)=\left(x_{1}(t), \ldots, x_{n}(t)\right)^{T} \in R^{n}$ and $u(t) \in R$ are the system state and input, respectively. $x_{2}(t), \ldots, x_{n}(t)$ are unmeasurable, $\bar{x}_{i}(t)=\left(x_{1}(t), \ldots, x_{i}(t)\right)^{T}, \bar{x}_{n}(t)=x(t) . d_{i}(t)$ : $R^{+} \rightarrow\left[0, d_{i}\right], i=1, \ldots, n$, are the time-varying delays satisfying $\dot{d}_{i}(t) \leq \vartheta_{i}<1$ for known constants $d_{i}$ and $\vartheta_{i}$; the system initial condition is $x(\theta)=\varphi_{0}(\theta), \theta \in[0, d]$ with $d=\max \left\{d_{1}, \ldots, d_{n}\right\} . p_{i} \in R_{\text {odd }}^{\geq 1}:=\{p / q \mid p$ and $q$ are positive odd integers, and $p \geq q\}, i=1, \ldots, n$, are said to be the high orders of the system. $f_{i}, i=1, \ldots, n$, are unknown continuous functions.

System (1) represents an important class of systems which can model many frequently met practical systems, such as the underactuated, weakly coupled, unstable mechanical system, and the cascade chemical system. However, the global stabilization of (1) has been widely recognized as difficulty because its Jacobian linearization, being neither controllable nor feedback linearizable, leads to the traditional design tools hardly applicable to such system. Mainly, thanks to the method of adding a power integrator, when $d_{i}(t)=0$, the 
state feedback stabilization of (1) has been well studied and a number of interesting results have been achieved over the last decades; for example, one can see [10-18] and the references therein.

However, when $d_{i}(t) \neq 0$, the global stabilization of (1) is much more challenging because trade-off of time-delay effect and identification of time-delay restriction. In this regard, some results were also reported. In [19], high-order time-delay nonlinear systems were first investigated, and, by imposed some restriction on the system growth, a continuous state feedback controller was given. Later, the authors in [2023] further relaxed conditions placed on the system growth and addressed state or output feedback stabilization problem for high-order nonlinear systems with multiple time delays, respectively.

Motivated by the continuous control ideas in $[11,18]$, this paper continues the investigations in [19-23] and further addresses the stabilizing control design for high-order timedelay nonlinear systems (1). The main contributions of this paper are twofolds. (i) By comparison with the existing results in $[19-21,23]$, the nonlinear growth condition is largely relaxed and a much weaker sufficient condition is given. (ii) By successfully overcoming some essential difficulties such as the weaker assumption on the system growth and the construction of a $C^{1}$, proper, and positive definite Lyapunov function, a new method to global stabilization of high-order time-delay nonlinear systems by state feedback is given and leads to much more general results than the previous ones.

The remainder of this paper is organized as follows. Section 2 presents some necessary notations, definition, and preliminary results. Section 3 formulates the control problem, presents the design scheme to the controller, and gives the main contributions of this paper. Section 4 gives a simulation example to demonstrate the effectiveness of the theoretical results. Section 5 addresses some concluding remarks. The paper ends with an appendix.

\section{Mathematical Preliminaries}

The following notations, definition, and lemmas will be used throughout the paper.

Notations. $R^{+}$denotes the set of all nonnegative real numbers and $R^{n}$ denotes the real $n$-dimensional space. $R_{\text {odd }}^{+}:=\{p / q \mid p$ and $q$ are positive odd integers $\}$ and $R_{\text {odd }}^{\geq 1}:=\{p / q \mid p$ and $q$ are positive odd integers, and $p \geq q$ \}. For a given vector $X$, $X^{T}$ denotes its transpose, and $|X|$ denotes its Euclidean norm. $C^{i}$ denotes the set of all functions with continuous $i$ th partial derivatives. $\mathscr{K}$ denotes the set of all functions: $R^{+} \rightarrow R^{+}$, which are continuous, strictly increasing, and vanishing at zero; $\mathscr{K}_{\infty}$ denotes the set of all functions which are of class $\mathscr{K}$ and unbounded. For any $a \in R^{+}$and $x \in R$, the function $[x]^{a}$ is defined as $[x]^{a}=\operatorname{sgn}(x)|x|^{a}$. Besides, let $\sum_{l=1}^{1} p_{1} p_{0}=1$ and the arguments of the functions (or the functionals) will be omitted or simplified, whenever no confusion can arise from the context. For instance, we sometimes denote a function $f(x(t))$ by simply $f(x), f(\cdot)$, or $f$.
Definition 1 (see [24]). Weighted homogeneity: for fixed coordinates $\left(x_{1}, \ldots, x_{n}\right) \in R^{n}$ and real numbers $r_{i}>0$, $i=1, \ldots, n$, consider the following.

(i) The dilation $\Delta_{\varepsilon}(x)$ is defined by $\Delta_{\varepsilon}(x)=$ $\left(\varepsilon^{r_{1}} x_{1}, \ldots, \varepsilon^{r_{n}} x_{n}\right)$ for any $\varepsilon>0$, where $r_{i}$ is called the weights of the coordinates. For simplicity, we define dilation weight $\Delta=\left(r_{1}, \ldots, r_{n}\right)$.

(ii) A function $V \in\left(R^{n}, R\right)$ is said to be homogeneous of degree $\tau$ if there is a real number $\tau \in R$ such that $V\left(\Delta_{\varepsilon}(x)\right)=\varepsilon^{\tau} V\left(x_{1}, \ldots, x_{n}\right)$ for any $x \in R^{n} \backslash\{0\}, \varepsilon>0$.

(iii) A vector field $f \in\left(R^{n}, R^{n}\right)$ is said to be homogeneous of degree $\tau$ if there is a real number $\tau \in R$ such that $f_{i}\left(\Delta_{\varepsilon}(x)\right)=\varepsilon^{\tau+r_{i}} f_{i}(x)$, for any $x \in R^{n} \backslash\{0\}, \varepsilon>0$, $i=1, \ldots, n$.

(iv) A homogeneous $p$-norm is defined as $\|x\|_{\Delta, p}=$ $\left(\sum_{i=1}^{n}\left|x_{i}\right|^{p / r_{i}}\right)^{1 / p}$ for all $x \in R^{n}$, for a constant $p \geq 1$. For simplicity, in this paper, we choose $p=2$ and write $\|x\|_{\Delta}$ for $\|x\|_{\Delta, 2}$.

Lemma 2 (see [24]). Given a dilation weight $\Delta=\left(r_{1}, \ldots, r_{n}\right)$, suppose $V_{1}(x)$ and $V_{2}(x)$ are homogeneous functions of degree $\tau_{1}$ and $\tau_{2}$, respectively. Then $V_{1}(x) V_{2}(x)$ is also homogeneous with respect to the same dilation weight $\Delta$. Moreover, the homogeneous degree of $V_{1}(x) V_{2}(x)$ is $\tau_{1}+\tau_{2}$.

Lemma 3 (see [24]). Suppose $V: R^{n} \rightarrow R$ is a homogeneous function of degree $\tau$ with respect to the dilation weight $\Delta$. Then the following holds:

(i) $\partial V / \partial x_{i}$ is homogeneous of degree $\tau-r_{i}$ with $r_{i}$ being the homogeneous weight of $x_{i}$;

(ii) there is a constant $c$ such that $V(x) \leq c\|x\|_{\Delta}^{\tau}$. Moreover, if $V(x)$ is positive definite; then $\underline{c}\|x\|_{\Delta}^{\tau} \leq V(x)$, where $\underline{c}$ is a constant.

Lemma 4 (see [25]). For $x \in R, y \in R$, and $p \geq 1$ being $a$ constant, the following inequalities hold:

$$
\begin{gathered}
|x+y|^{p} \leq 2^{p-1}\left|x^{p}+y^{p}\right| \\
(|x|+|y|)^{1 / p} \leq|x|^{1 / p}+|y|^{1 / p} \leq 2^{(p-1) / p}(|x|+|y|)^{1 / p} .
\end{gathered}
$$

If $p \geq 1$ is odd, then

$$
\begin{gathered}
|x-y|^{p} \leq 2^{p-1}\left|x^{p}-y^{p}\right| \\
\left|x^{1 / p}-y^{1 / p}\right| \leq 2^{(p-1) / p}(|x-y|)^{1 / p} .
\end{gathered}
$$

Lemma 5 (see [26]). Let $x$, $y$ be real variables; then for any positive real numbers $a, m$, and $n$, one has

$$
\begin{aligned}
a|x|^{m}|y|^{n} \leq & b|x|^{m+n} \\
& +\frac{n}{m+n}\left(\frac{m+n}{m}\right)^{-m / n} a^{(m+n) / n} b^{-m / n}|y|^{m+n},
\end{aligned}
$$

where $b>0$ is any real number. 


\section{State Feedback Controller Design}

3.1. Assumption. The following assumption is imposed on system (1) in this paper.

Assumption 6. For $i=1, \ldots, n$, there are constants $a>0$ and $\tau>-1 / \sum_{l=1}^{n} p_{1} \cdots p_{l-1}$ such that

$$
\begin{aligned}
& \left|f_{i}\left(\bar{x}_{i}(t), x_{1}\left(t-d_{1}(t)\right), \ldots, x_{i}\left(t-d_{i}(t)\right)\right)\right| \\
& \quad \leq a \sum_{j=1}^{i}\left(\left|x_{j}(t)\right|^{\left(r_{i}+\tau\right) / r_{j}}+\left|x_{j}\left(t-d_{j}(t)\right)\right|^{\left(r_{i}+\tau\right) / r_{j}}\right),
\end{aligned}
$$

where $r_{1}=1$ and $r_{i+1}=\left(r_{i}+\tau\right) / p_{i}>0, i=1, \ldots, n$.

For simplicity, it is assumed that $\tau=-m / n$ with $m$ being any even integer and $m$ being any odd integer, under which and the definition of $r_{i}$ in Assumption 6, we know that $r_{i} \in$ $R_{\text {odd }}^{+}$.

Remark 7. Assumption 6, which gives the nonlinear growth condition on the system drift terms, encompasses the assumptions in existing results [19-21, 23]. Specifically, when $d_{i}(t)=d$ and $\tau=0$, it reduces to Assumption 2.1 in [19]. When $\tau \in[0,+\infty)$, it is equivalent to those in $[20,21,23]$. Moreover, it is worth pointing out this assumption cannot be covered by Assumption 1 in [22], which can be represented as

$$
\begin{aligned}
& \left|f_{i}\left(\bar{x}_{i}(t), x_{1}\left(t-d_{1}(t)\right), \ldots, x_{i}\left(t-d_{i}(t)\right)\right)\right| \\
& \leq a \sum_{j=1}^{i}\left(\left|x_{j}(t)\right|^{\left(r_{i}+\tau\right) / r_{j}}+\left|x_{j}\left(t-d_{j}(t)\right)\right|^{\left(r_{i}+\tau\right) / r_{j}}\right) \\
& \quad+a\left(\left|x_{i}(t)\right|+\left|x_{j}\left(t-d_{j}(t)\right)\right|\right) \\
& \quad+a \sum_{j=1}^{i}\left(\left|x_{j}(t)\right|^{1 / p_{j} \cdots p_{i-1}}+\left|x_{j}\left(t-d_{j}(t)\right)\right|^{1 / p_{j} \cdots p_{i-1}}\right),
\end{aligned}
$$

where $\tau \in[0,+\infty)$ is a constant. For example, the simple system $\dot{x}_{1}=x_{2}+x_{1}^{3 / 5}(t-d), \dot{x}_{2}=u$ cannot be globally stabilized using the design method presented in [22] because of the presence of low-order term $x_{1}^{3 / 5}(t-d)$, but it is easy to verify that Assumption 6 in this paper is satisfied with $a=1$ and $\tau=-2 / 5 \in(-1 / 2,+\infty)$. This means that, to some extent, the system studied in this paper is less restrictive and allows for a much broader class of systems.

The objective of this paper is to design a state feedback controller for system (1) under Assumption 6 such that the closed-loop system is globally asymptotically stable.

To this end, we first introduce the following coordinate transformation:

$$
\begin{aligned}
z_{1}=x_{1}, \quad z_{i} & =\frac{x_{i}}{L^{\kappa_{i}}}, \quad i=2, \ldots, n, \\
v^{p_{n}} & =\frac{u^{p_{n}}}{L^{\kappa_{n}+1}},
\end{aligned}
$$

where $\kappa_{1}=0, \kappa_{i+1}=\left(\kappa_{i}+1\right) / p_{i}, i=1, \ldots, n-1$, and $L>1$ is a constant to be determined.

Then, under the new coordinates $z_{i}$ 's, system (1) is transformed into

$$
\begin{gathered}
\dot{z}_{i}=L x_{i+1}^{p_{i}}+\frac{f_{i}}{L^{\kappa_{i}}}, \quad i=1, \ldots, n-1 \\
\dot{z}_{n}=L v^{p_{n}}+\frac{f_{n}}{L^{\kappa_{n}}} .
\end{gathered}
$$

Remark 8. We need to emphasize that the gain $L$ creates an extra freedom in control design. As a matter of fact, in the proof of Theorem 10, complex uncertainties will inevitably be produced in the amplification of nonlinearities. Hence, the gain $L$ can be used to effectively dominate all the possible uncertainties.

3.2. State Feedback Controller Design for Nominal Nonlinear System. We first construct a state feedback controller for the nominal nonlinear system of (8):

$$
\begin{gathered}
\dot{z}_{i}=L z_{i+1}^{p_{i}}, \quad i=1, \ldots, n-1 \\
\dot{z}_{n}=L v^{p_{n}}
\end{gathered}
$$

Step 1. Let $\xi_{1}=z_{1}^{\sigma / r_{1}}$, where $\sigma \geq \max _{1 \leq i \leq n}\left\{1, \tau+r_{i}\right\}$ is a positive number, and choose the Lyapunov function

$$
V_{1}=W_{1}=\int_{z_{1}^{*}}^{z_{1}}\left(s^{\sigma / r_{1}}-z_{1}^{* \sigma / r_{1}}\right)^{\left(2 \sigma-\tau-r_{1}\right) / \sigma} d s
$$

with $z_{1}^{*}=0$. From (9), it follows that

$$
\dot{V}_{1} \leq-n L \xi_{1}^{2}+L \xi_{1}^{\left(2 \sigma-\tau-r_{1}\right) / \sigma}\left(z_{2}^{p_{1}}-z_{2}^{* p_{1}}\right)
$$

where the virtual controller is chosen as

$$
z_{2}^{*}=-n^{1 / p_{1}} z_{1}^{\left(r_{1}+\tau\right) / p_{1}}:=-\beta_{1}^{r_{2} / \sigma} \xi_{1}^{r_{2} / \sigma} .
$$

Step $i(i=2, \ldots, n)$. In this step, we can obtain the following property, whose proof is given in the appendix.

Proposition 9. Assume that at step $i-1$, there is a $C^{1}$, proper, and positive definite Lyapunov function $V_{i-1}$, and a set of virtual controllers $z_{1}^{*}, \ldots, z_{i}^{*}$ defined by

$$
\begin{array}{ccc}
z_{1}^{*} & =0, & \xi_{1}=z_{1}^{\sigma / r_{1}}-z_{1}^{* \sigma / r_{1}} \\
z_{2}^{*}=-\beta_{1}^{r_{2} / \sigma} \xi_{1}^{r_{2} / \sigma}, & \xi_{2}=z_{2}^{\sigma / r_{2}}-z_{2}^{* \sigma / r_{2}} \\
\vdots & \vdots \\
z_{i}^{*}=-\beta_{i-1}^{r_{i} / \sigma} \xi_{i-1}^{r_{i} / \sigma}, & \xi_{i}=z_{i}^{\sigma / r_{i}}-z_{i}^{* \sigma / r_{i}}
\end{array}
$$

with $\beta_{1}>0, \ldots, \beta_{i-1}>0$ being constants, such that

$$
\begin{aligned}
\dot{V}_{i-1} \leq & -(n-i+2) L \sum_{j=1}^{i-1} \xi_{j}^{2} \\
& +\xi_{i-1}^{\left(2 \sigma-\tau-r_{i-1}\right) / \sigma}\left(z_{i}^{p_{i-1}}-z_{i}^{* p_{i-1}}\right) .
\end{aligned}
$$


Then the ith Lyapunov function defined by

$$
\begin{gathered}
V_{i}\left(\bar{z}_{i}\right)=V_{i-1}\left(\bar{z}_{i-1}\right)+W_{i}\left(\bar{z}_{i}\right) \\
W_{i}\left(\bar{z}_{i}\right)=\int_{z_{i}^{*}}^{z_{i}}\left(s^{\sigma / r_{i}}-z_{i}^{* \sigma / r_{i}}\right)^{\left(2 \sigma-\tau-r_{i}\right) / \sigma} d s
\end{gathered}
$$

is $C^{1}$, proper, and positive definite, and there is $z_{i+1}^{*}=$ $-\beta_{i}^{r_{i+1} / \sigma} \xi_{i}^{r_{i+1} / \sigma}$ such that

$$
\dot{V}_{i} \leq-(n-i+1) L \sum_{j=1}^{i} \xi_{j}^{2}+L \xi_{i}^{\left(2 \sigma-\tau-r_{i}\right) / \sigma}\left(z_{i+1}^{p_{i}}-z_{i+1}^{* p_{i}}\right) .
$$

Hence at step $n$, choosing

$$
\begin{aligned}
& V_{n}=\sum_{i=1}^{n} \int_{z_{i}^{*}}^{z_{i}}\left(s^{\sigma / r_{i}}-z_{i}^{* \sigma / r_{i}}\right)^{\left(2 \sigma-\tau-r_{i}\right) / \sigma} d s \\
& v=-\beta_{n}^{r_{n+1} / \sigma} \xi_{n}^{r_{n+1} / \sigma}=-\left(\sum_{i=1}^{n} \bar{\beta}_{i} z_{i}^{\sigma / r_{i}}\right)^{r_{n+1} / \sigma}
\end{aligned}
$$

from Proposition 9, we arrive at

$$
\dot{V}_{n} \leq-L \sum_{j=1}^{n} \xi_{j}^{2}
$$

where $\xi_{i}=z_{i}^{\sigma / r_{i}}-z_{i}^{* \sigma / r_{i}}$ and $\bar{\beta}_{i}=\beta_{n} \cdots \beta_{i} i-1, \ldots, n$ are positive constant. The system (8) and (17) can be written as the following compact form:

$$
\dot{z}=L E(z)+F(z)
$$

where $z=\left(z_{1}, \ldots, z_{n}\right)^{T}, E(z)=\left(z_{2}^{p_{1}}, \ldots, z_{n}^{p_{n-1}}, v^{p_{n}}\right)^{T}$, and $F(z)=\left(f_{1}, f_{2} / L^{\kappa_{2}}, \ldots, f_{n} / L^{\kappa_{n}}\right)^{T}$. Introducing the dilation weight $\Delta=\left(r_{1}, \ldots, r_{n}\right)$, from Definition 1 , it can be shown that $V_{n}$ is homogeneous of degree $2 \sigma-\tau$ with respect to $\Delta$.

3.3. Stability Analysis. We state the main results in this paper.

Theorem 10. For the time-delay nonlinear system (1) under Assumption 6, the state feedback controller $u^{p_{n}}=L^{\kappa_{n}+1} v^{p_{n}}$ in (7) and (17) renders that the equilibrium at the origin of the closed-loop system is globally asymptotically stable.

Proof. We prove Theorem 10 by four steps.

Step 1. We first prove that $u^{p_{n}}$ preserves the equilibrium at the origin.

From (17) and $r_{n+1} p_{n}=r_{n}+\tau$, we have

$$
v^{p_{n}}(z)=-\left(\sum_{i=1}^{n} \bar{\beta}_{i} z_{i}^{\sigma / r_{i}}\right)^{\left(r_{n}+\tau\right) / \sigma}
$$

By which and the definitions of $r_{i}$ 's and $\sigma$, we easily see that $u^{p_{n}}=L^{\kappa_{n}+1} v^{p_{n}}$ is a continuous function of $z$ and $u^{p_{n}}(z)=0$ for $z=0$. This together with Assumption 6 implies that the solutions of $z$-system is defined on a time interval $\left[-d, t_{M}\right)$, where $t_{M}>0$ may be a finite constant or $+\infty$, and $u^{p_{n}}$ preserves the equilibrium at the origin.

Step 2. We construct a Lyapunov-Krasovskii functional

$$
V(z(t))=V_{n}(z(t))+\sum_{i=1}^{n} \frac{\lambda}{1-\vartheta_{i}} \int_{t-d_{i}(t)}^{t}\|z(\varepsilon)\|_{\Delta}^{2 \sigma} d \varepsilon,
$$

where $\lambda$ is a positive parameter to be determined later. Because $V_{n}(z(t))$ is $C^{1}$, positive definite radially unbounded and by Lemma 4.3 in [27], there exist two class $\mathscr{K}_{\infty}$ functions $\beta_{1}$ and $\beta_{21}$ such that

$$
\beta_{1}(|z(t)|) \leq V_{n}(z(t)) \leq \beta_{21}(|z(t)|) .
$$

According to the homogeneous theory, there are positive constants $\underline{c}$ and $\bar{c}$ such that

$$
\underline{c}\|z(t)\|_{\Delta}^{2 \sigma} \leq W(z(t)) \leq \bar{c}\|z(t)\|_{\Delta}^{2 \sigma}
$$

where $W(z(t))$ is a positive definite function, whose homogeneous degree is $2 \sigma$. Therefore, the following inequality holds:

$$
\underline{\beta}_{22}(|z(t)|) \leq W(z(t)) \leq \bar{\beta}_{22}(|z(t)|)
$$

with two class $\mathscr{K}_{\infty}$ functions $\underline{\beta}_{22}$ and $\bar{\beta}_{22}$.

With the help of $d_{i}(t): \bar{R}^{+} \rightarrow\left[0, d_{i}\right], i=1, \ldots, n$, it follows that

$$
\begin{aligned}
& \sum_{i=1}^{n} \frac{\lambda}{1-\vartheta_{i}} \int_{t-d_{i}(t)}^{t}\|z(\varepsilon)\|_{\Delta}^{2 \sigma} d \varepsilon \\
& \quad \leq \widehat{c}_{i} \int_{t-d_{i}}^{t} \widetilde{\beta}_{22}|z(\varepsilon)| d \varepsilon \\
& \quad \leq \widehat{c}_{i} \int_{-d_{i}}^{0} \widetilde{\beta}_{22}(|z(s+t)|) d(s+t) \\
& \quad \leq \widetilde{c}_{i} \sup _{-d_{i} \leq s \leq 0} \widetilde{\beta}_{22}(|z(s+t)|) \\
& \quad \leq \beta_{22}\left(\sup _{-d_{i} \leq s \leq 0}|z(s+t)|\right)
\end{aligned}
$$

where $\widetilde{\beta}_{22}$ and $\beta_{22}$ are class $\mathscr{K}_{\infty}$ functions and $\widehat{c}_{i}$ and $\widetilde{c}_{i}, i=1, \ldots, n$, are positive constants, because $|z(t)| \leq$ $\sup _{-d \leq s \leq 0}|z(s+t)|$ and $\sup _{-d_{i} \leq s \leq 0}|z(s+t)| \leq \sup _{-d \leq s \leq 0}|z(s+t)|$. Defining $\beta_{2}=\beta_{21}+\beta_{22}$, from (21), (22), and (25), it follows that

$$
\beta_{1}(|z(t)|) \leq V(z(t)) \leq \beta_{2}\left(\sup _{-d \leq s \leq 0}|z(s+t)|\right) .
$$

Step 3. Because $V_{n}(z)$ and $E(z)$ are homogeneous of degree $2 \sigma-\tau$ and $\tau$ with respect to $\Delta$, by Lemmas 2 and 3 , there is a constant $c_{1}$ such that

$$
\frac{\partial V_{n}(z)}{\partial z} L E(z) \leq-c_{1} L\|z(t)\|_{\Delta}^{2 \sigma} .
$$


By (7), Assumption 6 and $L>1$, we can find constants $\delta_{i}>0$ and $0<v_{i} \leq 1$ such that

$$
\begin{aligned}
\left|\frac{f_{i}(\cdot)}{L^{\kappa_{i}}}\right| \leq & \frac{a}{L^{\kappa_{i}}} \sum_{j=1}^{i}\left(\left|x_{j}(t)\right|^{\left(r_{i}+\tau\right) / r_{j}}+\left|x_{j}\left(t-d_{j}(t)\right)\right|^{\left(r_{i}+\tau\right) / r_{j}}\right) \\
= & a \sum_{j=1}^{i} L^{\kappa_{j}\left(r_{i}+\tau\right) / r_{j}-\kappa_{i}} \\
& \times\left(\left|z_{j}(t)\right|^{\left(r_{i}+\tau\right) / r_{j}}+\left|z_{j}\left(t-d_{j}(t)\right)\right|^{r_{i}+\tau / r_{j}}\right) \\
\leq & \delta_{i} L^{1-v_{i}}\left(\|z(t)\|_{\Delta}^{r_{i}+\tau}+\sum_{j=1}^{i}\left\|z\left(t-d_{j}(t)\right)\right\|_{\Delta}^{r_{i}+\tau}\right) ;
\end{aligned}
$$

since it can be seen that by definition $r_{j}=\tau \kappa_{j}+1 /\left(p_{1} \cdots p_{j-1}\right)$, so

$$
\begin{aligned}
& \frac{\kappa_{j}\left(r_{i}+\tau\right)}{r_{j}}-\kappa_{i} \\
& =\frac{\kappa_{j}\left(\tau \kappa_{i}+1 /\left(p_{1} \cdots p_{i-1}\right)+\tau\right)}{\tau \kappa_{j}+1 /\left(p_{1} \cdots p_{j-1}\right)}-\kappa_{i} \\
& =\frac{\tau \kappa_{j}-\left(\kappa_{j}-\kappa_{i}\right) /\left(p_{1} \cdots p_{i-1}\right)}{\tau \kappa_{j}+1 /\left(p_{1} \cdots p_{j-1}\right)} \\
& \left\{\begin{array}{c}
\leq \frac{\tau \kappa_{j}}{\tau \kappa_{j}+1 /\left(p_{1} \cdots p_{j-1}\right)}, \\
\tau \geq 0 \\
=\frac{\tau \kappa_{j}-\left(\kappa_{j}-\kappa_{i}\right) /\left(p_{1} \cdots p_{i-1}\right)}{\left(\tau \sum_{l=1}^{j} p_{1} \cdots p_{l-2}+1\right) /\left(p_{1} \cdots p_{j-1}\right)}, \\
0>\tau>\frac{-1}{\left(\sum_{l=1}^{n} p_{1} \cdots p_{l-1}\right)}
\end{array}\right. \\
& \left\{\begin{array}{ll}
<1, & \tau \geq 0 \\
\leq 0, & 0>\tau>\frac{-1}{\left(\sum_{l=1}^{n} p_{1} \cdots p_{l-1}\right)}
\end{array} .\right.
\end{aligned}
$$

Noting that for $i=1, \ldots, n, \partial V_{n}(z) / \partial z_{i}$ is homogeneous of degree $2 \sigma-\tau-r_{i}$; from Lemma 5 , we obtain

$$
\begin{aligned}
\left|\frac{\partial V_{n}(z)}{\partial z} F(z)\right| \leq & \sum_{i=1}^{n}\left|\frac{\partial V_{n}(z)}{\partial z_{i}}\right|\left|\frac{f_{i}(\cdot)}{L^{\kappa_{i}}}\right| \\
\leq & \sum_{i=1}^{n} \rho_{i 1} L^{1-v_{0}}\|z(t)\|_{\Delta}^{2 \sigma} \\
& +\sum_{i=1}^{n} \rho_{i 2} L^{1-v_{0}}\left\|z\left(t-d_{i}(t)\right)\right\|_{\Delta}^{2 \sigma}
\end{aligned}
$$

$$
\begin{aligned}
\leq & \rho_{1} L^{1-v_{0}}\|z(t)\|_{\Delta}^{2 \sigma} \\
& +\rho_{2} L^{1-v_{0}} \sum_{i=1}^{n}\left\|z\left(t-d_{i}(t)\right)\right\|_{\Delta}^{2 \sigma},
\end{aligned}
$$

where $\rho_{i 1}, \rho_{i 2}, i=1, \ldots, n, \rho_{1}=\sum_{i=1}^{n} \rho_{i 1}, \rho_{2}=\max _{1 \leq i \leq n}\left\{\rho_{i 2}\right\}$, and $\nu_{0}=\min _{1 \leq i \leq n}\left\{v_{i}\right\} \leq 1$ are positive constants.

According to (19), (21), (27), and (30), we get

$$
\begin{aligned}
\dot{V}= & \frac{\partial V_{n}(z)}{\partial z} L E(z)+\frac{\partial V_{n}(z)}{\partial z} F(z) \\
& +\sum_{i=1}^{n} \frac{\lambda}{1-\vartheta_{i}}\|z(t)\|_{\Delta}^{2 \sigma}-\sum_{i=1}^{n} \lambda\left\|z\left(t-d_{i}(t)\right)\right\|_{\Delta}^{2 \sigma} \\
\leq & -\left(c_{1} L-\rho_{1} L^{1-v_{0}}-\rho_{2} L^{1-v_{0}} \sum_{i=1}^{n} \frac{1}{1-\vartheta_{i}}\right)\|Z(t)\|_{\Delta}^{2 \sigma} \\
& -\left(\lambda-\rho_{2} L^{1-\nu_{0}}\right) \sum_{i=1}^{n}\left\|Z\left(t-d_{i}(t)\right)\right\|_{\Delta}^{2 \sigma} .
\end{aligned}
$$

Therefore, by choosing

$$
\begin{gathered}
\lambda=\rho_{2} L^{1-\nu_{0}}, \\
L>\max \left\{\left(\frac{\rho_{1}+\rho_{2} \sum_{i=1}^{n} 1 /\left(1-\vartheta_{i}\right)}{c_{1}}\right)^{1 / \nu_{0}}, 1\right\},
\end{gathered}
$$

there exists a constant $c^{*}$ such that

$$
\dot{V} \leq-c^{*}\|z(t)\|_{\Delta}^{2 \sigma}
$$

from which, (23), and (24), we have

$$
\dot{V} \leq-\beta_{3}(|z(t)|)
$$

for a class $\mathscr{K}_{\infty}$ function $\beta_{3}$.

By (26) and (34), we immediately get $t_{M}=+\infty$. Therefore, all solutions of $z$-system are defined on a time interval $[-d,+\infty)$. Then by Lyapunov-Krasovskii stability theorem [1], we can conclude that $\lim _{t \rightarrow \infty} z(t)=0$.

Step 4. Since (7) is an equivalent transformation, the closedloop system consisting of (1), $u^{p_{n}}=L^{\kappa_{n}+1} v^{p_{n}}$ in (7) and (17), has the same properties as the system (8) and (17). Thus, the proof is completed.

3.4. Extension. In this subsection, we can extend the results developed above to high-order time-delay nonlinear system in nontriangular form:

$$
\begin{aligned}
& \dot{x}_{i}(t) \\
& =x_{i+1}^{p_{i}}(t)+f_{i}\left(\bar{x}_{n}(t), x_{1}\left(t-d_{1}(t)\right), \ldots, x_{n}\left(t-d_{n}(t)\right)\right) \\
& \dot{x}_{n}(t) \\
& =u^{p_{n}}(t)+f_{n}\left(\bar{x}_{n}(t), x_{1}\left(t-d_{1}(t)\right), \ldots, x_{n}\left(t-d_{n}(t)\right)\right)
\end{aligned}
$$

under the following assumption. 
Assumption 11. For $i=1, \ldots, n$, there are constants $a>0$, $L>1,0<v_{i} \leq 1$, and $\tau>-1 / \sum_{l=1}^{n} p_{1} \cdots p_{l-1}$ such that

$$
\begin{aligned}
\left|\frac{f_{i}(\cdot)}{L^{\kappa_{i}}}\right| \leq & a L^{1-v_{i}} \\
& \times \sum_{j=1}^{n}\left(\left|\frac{x_{j}(t)}{L^{\kappa_{j}}}\right|^{\left(r_{i}+\tau\right) / r_{j}}+\left|\frac{x_{j}\left(t-d_{j}(t)\right)}{L^{\kappa_{j}}}\right|^{\left(r_{i}+\tau\right) / r_{j}}\right),
\end{aligned}
$$

where $\kappa_{1}=0, r_{1}=1, \kappa_{i+1}=\left(\kappa_{i}+1\right) / p_{i}$, and $r_{i+1}=\left(r_{i}+\tau\right) / p_{i}>$ $0, i=1, \ldots, n$.

It is obvious that Assumption 6 is a special case of Assumption 11, and, under this, some more general results are given.

Theorem 12. For the time-delay nonlinear system (35) under Assumption 11, the state feedback controller $u^{p_{n}}=L^{\kappa_{n}+1} v^{p_{n}}$ in (7) and (17) renders that the equilibrium at the origin of the closed-loop system is globally asymptotically stable.

Proof. Similar to (28), Assumption 11 will directly lead to (30). The rest of the proof is similar to that of Theorem 10 and hence is omitted here.

\section{Simulation Example}

To illustrate the effectiveness of the proposed controller, we consider the following low-dimensional system:

$$
\begin{gathered}
\dot{x}_{1}=x_{2}^{9 / 7}+\frac{1}{4} x_{2}^{9 / 11}\left(t-d_{1}(t)\right) \\
\dot{x}_{2}=u^{3}+\frac{1}{8} \ln \left(1+x_{2}^{2}\right)+\frac{1}{12} x_{2}^{1 / 4}\left(t-d_{2}(t)\right) \sin x_{2},
\end{gathered}
$$

where $d_{1}(t)=1 / 5(1+\sin (t)), p_{1}=9 / 7$, and $p_{2}=3$.

It is worth pointing out that system (37) cannot be globally stabilized even by state feedback, using the design methods presented in [19-23] because of the presence of low-order term $(1 / 4) x_{1}^{9 / 11}\left(t-d_{1}(t)\right)$. Choose $\tau=-2 / 11 \epsilon$ $(-3 / 8,+\infty)$, then $r_{1}=1, r_{2}=\left(r_{1}+\tau\right) / p_{1}=7 / 11$ and $r_{3}=\left(r_{2}+\tau\right) / p_{2}=5 / 33$. By Lemma 5 , it can be verified that $\left|f_{1}\right| \leq(1 / 4)\left|x_{1}\left(t-d_{1}(t)\right)\right|^{9 / 11}$ and $\left|f_{2}\right| \leq$ $(1 / 4)\left(\left|x_{2}\right|^{5 / 7}+\left|x_{2}\left(t-d_{2}(t)\right)\right|^{5 / 7}\right)$ satisfy Assumption 6 with $a=1 / 4$. Moreover, noting that $0 \leq d_{1}(t) \leq 1 / 5$ and $\dot{d}_{1}(t)=$ $1 / 5 \cos t \leq 1 / 5<1$, the controller proposed in this paper is applicable. Thus, in terms of the design steps developed in Section 3, a continuous controller of system (37) can be given to ensure that the closed-loop system meets the conclusions of Theorem 10.

Let $\sigma=1$ and the initial states be $x_{1}(\theta)=0.5, x_{2}(\theta)=$ -0.3 , and $\theta \in[-0.5,0]$. Using MATLAB, Figures 1 and 2 are obtained to exhibit the the trajectories of the closed-loop system states and the control input. From these figures, it can be seen that $x_{1}$ and $x_{2}$ are asymptotically regulated to zero, which demonstrates the effectiveness of the control method proposed in this paper.

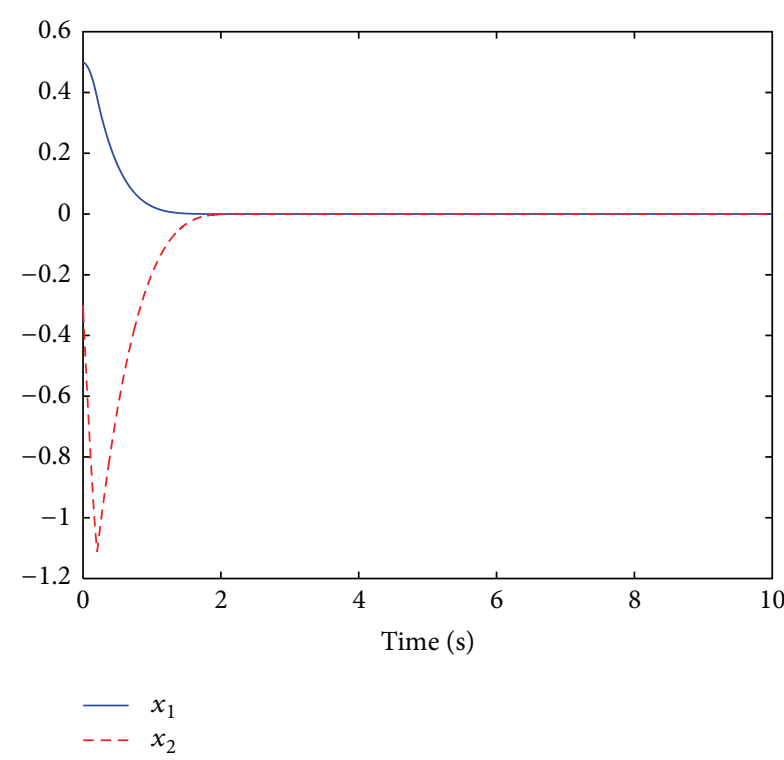

FIGURE 1: The trajectories of system states.

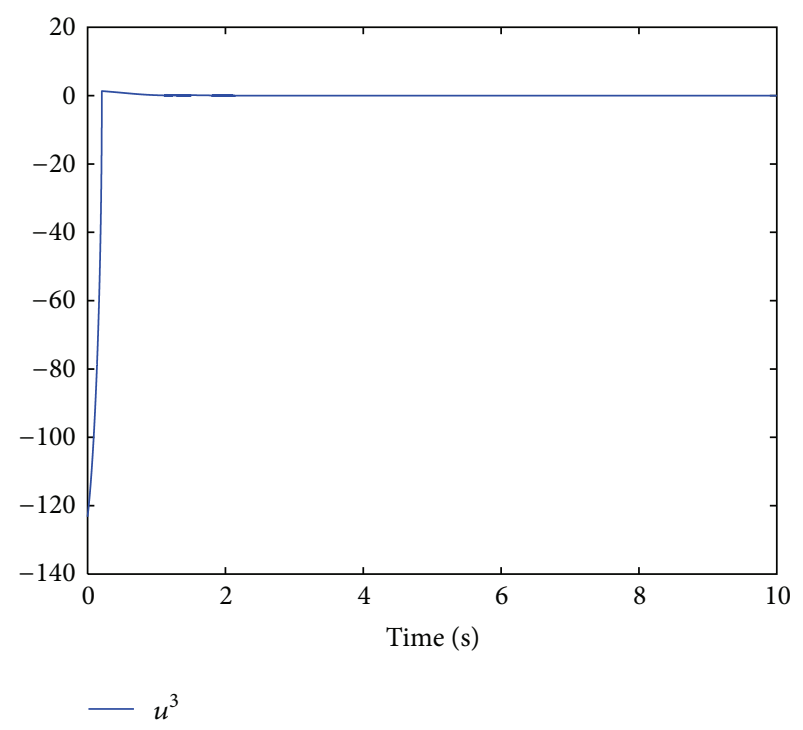

FIGURE 2: The trajectory of control input.

\section{Conclusion}

In this paper, a state feedback stabilization controller independent of time-delays is presented for a class of high-order nonlinear systems with time-varying delays under a weaker condition. The controller designed preserves the equilibrium at the origin and guarantees the globally asymptotic stability of the system. It should be noted that the proposed controller can only work well when the whole state vector is measurable. Therefore, a natural and more interesting problem is how to design output feedback stabilization controller for the systems studied in the paper if only partial state vector is measurable. In addition, in recent years, many results on stochastic nonlinear systems have been achieved [28-36], and 
so forth; an important problem is whether the results in this paper can be extended to stochastic high-order nonlinear systems.

\section{Appendix}

\section{Proof of Proposition 9}

We first prove that $V_{i}$ defined in (15) is $C^{1}$. According to the definition of $\sigma$, it is deduced that $\left(2 \sigma-\tau-r_{i}\right) / \sigma \geq 1$. From this, we know that $W_{i}$ is $C^{1}$, and then $V_{i}$ is also $C^{1}$.

Next, we prove that $V_{i}$ is positive definite and proper. When $z_{i}^{*} \leq z_{i}$, using $|x-y|^{p} \leq 2^{p-1}\left|x^{p}-y^{p}\right|$, this leads to

$$
\begin{aligned}
W_{i} & =\int_{z_{i}^{*}}^{z_{i}}\left(s^{\sigma / r_{i}}-z_{i}^{* \sigma / r_{i}}\right)^{\left(2 \sigma-\tau-r_{i}\right) / \sigma} d s \\
& \geq \frac{2^{\left(2 \sigma-\tau-r_{i}\right) / \sigma}}{2^{\left(2 \sigma-\tau-r_{i}\right) / r_{i}}} \int_{z_{i}^{*}}^{z_{i}}\left(s-z_{i}^{*}\right)^{\left(2 \sigma-\tau-r_{i}\right) / r_{i}} d s \\
& \geq \frac{r_{i} 2^{\left(2 \sigma-\tau-r_{i}\right) / \sigma}}{(2 \sigma-\tau) 2^{\left(2 \sigma-\tau-r_{i}\right) / r_{i}}}\left(z_{i}-z_{i}^{*}\right)^{(2 \sigma-\tau) / r_{i}} .
\end{aligned}
$$

Similarly, it can be shown that (A.1) also holds when $z_{i}^{*} \geq$ $z_{i}$. Therefore

$$
V_{i}=V_{i-1}+W_{i} \geq V_{i-1}+m_{i}\left|z_{i}-z_{i}^{*}\right|^{(2 \sigma-\tau) / r_{i}}
$$

which implies that $V_{i}$ is positive definite and proper, where $m_{i}>0$ is a constant.

At last, we prove inequality (16) by induction. We observe from (9), (14), and (15) that

$$
\begin{aligned}
\dot{V}_{i} \leq & -(n-i+2) L \sum_{j=1}^{i-1} \xi_{j}^{2}+L\left[\xi_{i}\right]^{\left(2 \sigma-\tau-r_{i}\right) / \sigma}\left(z_{i+1}^{p_{i}}-z_{i+1}^{* p_{i}}\right) \\
& -L\left[\xi_{i-1}\right]^{\left(2 \sigma-\tau-r_{i}\right) / \sigma} z_{i+1}^{* p_{i}} \\
& +L\left[\xi_{i-1}\right]^{\left(2 \sigma-\tau-r_{i-1}\right) / \sigma}\left(z_{i}^{p_{i-1}}-z_{i}^{* p_{i-1}}\right)+L \sum_{j=1}^{i-1} \frac{\partial W_{i}}{\partial z_{j}} z_{j+1}^{p_{j}} .
\end{aligned}
$$

We now estimate the last two terms on the right-hand side of inequality (A.3). obtains

Noting that $r_{i} p_{i-1} \leq 1$, using (13) and Lemma 4, one

$$
\left|z_{i}^{p_{i-1}}-z_{i}^{* p_{i-1}}\right| \leq 2^{\left(\sigma-r_{i} p_{i-1}\right) / \sigma}\left|\xi_{i}\right|^{r_{i} p_{i-1} / \sigma} .
$$

Using the fact $p_{i-1} r_{i}=r_{i-1}+\tau$ and (A.4), it follows from Lemma 5 that

$$
\begin{aligned}
& {\left[\xi_{i-1}\right]^{\left(2 \sigma-\tau-r_{i-1}\right)}\left(z_{i}^{p_{i-1}}-z_{i}^{* p_{i-1}}\right)} \\
& \quad \leq 2^{\left(\sigma-r_{i} p_{i-1}\right) / \sigma}\left|\xi_{i-1}\right|^{\left(2 \sigma-\tau-r_{i-1}\right) / \sigma}\left|\xi_{i}\right|^{r_{i} p_{i-1} / \sigma} \\
& \quad \leq \frac{1}{2} \xi_{i-1}^{2}+c_{i 1} \xi_{i}^{2},
\end{aligned}
$$

where $c_{i 1}$ is a positive constant.
With the help of (9), (13), and Lemmas 4 and 5, one has

$$
\begin{aligned}
& \sum_{j=1}^{i-1} \frac{\partial W_{i}}{\partial z_{j}} z_{j+1}^{p_{j}} \\
& =\sum_{j=1}^{i-1}\left(-\frac{2 \sigma-\tau-r_{i}}{\sigma} \frac{\partial\left(z_{i}^{* \sigma / r_{i}}\right)}{\partial z_{j}}\right. \\
& \left.\quad \times \int_{z_{i}^{*}}^{z_{i}}\left(s^{\sigma / r_{i}}-z_{i}^{* \sigma / r_{i}}\right)^{\left(\sigma-\tau-r_{i}\right) / \sigma} d s\right) z_{j+1}^{p_{j}} \\
& \leq b_{i} \sum_{j=1}^{i-1}\left|\xi_{i}\right|^{(\sigma-\tau) / \sigma}\left|z_{j}\right|^{\left(\sigma-r_{j}\right) / r_{j}}\left|z_{j+1}\right|^{p_{j}} \\
& \leq b_{i} \sum_{j=1}^{i-1}\left|\xi_{i}\right|^{(\sigma-\tau) / \sigma}\left(\left|\xi_{j}-\beta_{j-1} \xi_{j-1}\right|^{r_{j} / \sigma}\right)^{\left(\sigma-r_{j}\right) / r_{j}} \\
& \quad \times\left(\left|\xi_{j+1}-\beta_{j} \xi_{j}\right|^{r_{j+1} / \sigma}\right)^{p_{j}} \\
& \leq \widetilde{b}_{i} \sum_{j=1}^{i-1}\left|\xi_{i}\right|^{(\sigma-\tau) / \sigma}\left(\left|\xi_{j}\right|^{\left.+\beta_{j-1}\left|\xi_{j-1}\right|\right)^{\left(\sigma-r_{j}\right) / \sigma}}\right. \\
& \quad \times\left(\left|\xi_{j+1}\right|+\beta_{j}\left|\xi_{j}\right|\right)^{\left(r_{j}+\tau\right) / \sigma} \\
& \leq \frac{1}{2} \sum_{j=1}^{i-1} \xi_{j}^{2}+c_{i 2} \xi_{i}^{2},
\end{aligned}
$$

where $b_{i}, \widetilde{b}_{i}$, and $c_{i 2}$ are positive constants.

Choosing $z_{i+1}^{*}=-\beta_{i}^{r_{i+1} / \sigma}\left[\xi_{i}\right]^{r_{i+1} / \sigma}$ and $\beta_{i}=(n-i+1+$ $\left.c_{i 1}+c_{i 2}\right)^{\sigma / r_{i+1} p_{i}}$ and substituting (A.5), (A.6) into (A.3), one completes the proof.

\section{Conflict of Interests}

The authors declare that there is no conflict of interests regarding the publication of this paper.

\section{Acknowledgments}

The authors thank the editor and the anonymous reviewers for their constructive comments and suggestions for improving the quality of the paper. This work is partially supported by National Nature Science Foundation of China under Grant 61073065 and the Key Program of Science Technology Research of Education Department of Henan Province under Grants 13A120016, 14A520003.

\section{References}

[1] K. Gu, V. L. Kharitonov, and J. Chen, Stability of Time-Delay Systems, Birkhäuser, Berlin, Germany, 2003.

[2] Q. C. Zhong, Robust Control of Time-Delay Systems, Springer, London, UK, 2006. 
[3] L. Wu, X. Su, P. Shi, and J. Qiu, "A new approach to stability analysis and stabilization of discrete-time T-S fuzzy timevarying delay systems," IEEE Transactions on Systems, Man, and Cybernetics B, vol. 41, no. 1, pp. 273-286, 2011.

[4] X. Su, P. Shi, L. Wu, and S. Nguang, "Induced $\mathrm{l}_{2}$ filtering of fuzzy stochastic systems with time-varying delays," IEEE Transactions on Cybernetics, vol. 43, no. 4, pp. 1251-1264, 2013.

[5] X. Su, P. Shi, L. Wu, and Y. Song, "A novel control design on discrete-time takagi-sugeno fuzzy systems with time-varying delays," IEEE Transactions on Fuzzy Systems, vol. 21, no. 4, pp. 655-671, 2013.

[6] X. Su, L. Wu, P. Shi, and M. V. Basin, "Reliable filtering with strict dissipativity for TS fuzzy time-delay systems," IEEE Transactions on Cybernetics, 2014.

[7] X. Su, L. Wu, P. Shi, and Y. Song, "Ho model reduction of Takagi-Sugeno fuzzy stochastic systems," IEEE Transactions on Systems, Man, and Cybernetics B Cybernetics, vol. 42, no. 6, pp. 1574-1585, 2012.

[8] M. T. Alrifai, M. Zribi, M. Rayan, and M. S. Mahmoud, "On the control of time delay power systems," International Journal of Innovative Computing, Information and Control, vol. 9, no. 2, pp. 769-792, 2013.

[9] C. Onat, "A new concept on PI design for time delay systems: weighted geometrical center," International Journal of Innovative Computing, Information and Control, vol. 9, no. 4, pp. 15391556, 2013.

[10] W. Lin and C. Qian, "Adding one power integrator: a tool for global stabilization of high-order lower-triangular systems," Systems \& Control Letters, vol. 39, no. 5, pp. 339-351, 2000.

[11] C. Qian and W. Lin, "A continuous feedback approach to global strong stabilization of nonlinear systems," IEEE Transactions on Automatic Control, vol. 46, no. 7, pp. 1061-1079, 2001.

[12] W. Lin and C. Qian, "Adaptive control of nonlinearly parameterized systems: a nonsmooth feedback framework," IEEE Transactions on Automatic Control, vol. 47, no. 5, pp. 757-774, 2002.

[13] W. Lin and C. Qian, "Adaptive control of nonlinearly parameterized systems: the smooth feedback case," IEEE Transactions on Automatic Control, vol. 47, no. 8, pp. 1249-1266, 2002.

[14] J. Polendo and C. Qian, "An expanded method to robustly stabilize uncertain nonlinear systems," Communications in Information and Systems, vol. 8, no. 1, pp. 55-70, 2008.

[15] Z. Sun and Y. Liu, "Adaptive state-feedback stabilization for a class of high-order nonlinear uncertain systems," Automatica, vol. 43, no. 10, pp. 1772-1783, 2007.

[16] Z. Sun and Y. Liu, "Adaptive stabilisation for a large class of high-order uncertain non-linear systems," International Journal of Control, vol. 82, no. 7, pp. 1275-1287, 2009.

[17] J. Zhang and Y. Liu, "A new approach to adaptive control design without overparametrization for a class of uncertain nonlinear systems," Science China: Information Sciences, vol. 54, no. 7, pp. 1419-1429, 2011.

[18] X. Zhang and X. Xie, "Global state feedback stabilisation of nonlinear systems with high-order and low-order nonlinearities," International Journal of Control, vol. 87, no. 3, pp. 642-652, 2014.

[19] Z. Sun, Y. Liu, and X. Xie, "Global stabilization for a class of high-order time-delay nonlinear systems," International Journal of Innovative Computing, Information and Control, vol. 7, no. 12, pp. 7119-7130, 2011.

[20] Z. Sun, X. Xie, and Z. Liu, "Global stabilisation of high-order nonlinear systems with multiple time delays," International Journal of Control, vol. 86, no. 5, pp. 768-778, 2013.
[21] Z. Sun, X. Zhang, and X. Xie, "Continuous global stabilisation of high-order time-delay nonlinear systems," International Journal of Control, vol. 86, no. 6, pp. 994-1007, 2013.

[22] Z. Sun, Z. Liu, and X. Zhang, "New results on global stabilization for time-delay nonlinear systems with low order and highorder growth conditions," International Journal of Robust and Nonlinear Control, 2013.

[23] F. Gao, Y. Wu, and F. Yuan, "Global output feedback stabilization of high-order nonlinear systems with multiple time-varying delays," International Journal of Systems Science. In press.

[24] L. Rosier, "Homogeneous Lyapunov function for homogeneous continuous vector fields," Systems \& Control Letters, vol. 19, no. 6, pp. 467-473, 1992.

[25] J. Polendo and C. Qian, "A generalized homogeneous domination approach for global stabilization of inherently nonlinear systems via output feedback," International Journal of Robust and Nonlinear Control, vol. 17, no. 7, pp. 605-629, 2007.

[26] B. Yang and W. Lin, "Nonsmooth output feedback design with a dynamic gain for uncertain systems with strong nonlinearity," in Proceedings of the 46th IEEE Conference on Decision and Control (CDC '07), pp. 3495-3500, New Orieans , La, USA, December 2007.

[27] H. K. Khalil, Nonlinear Systems, Prentice-Hall, Upper Saddle River, NJ, USA, 1996.

[28] X. Xie and J. Tian, "State-feedback stabilization for high-order stochastic nonlinear systems with stochastic inverse dynamics," International Journal of Robust and Nonlinear Control, vol. 17, no. 14, pp. 1343-1362, 2007.

[29] X. Xie and J. Tian, "Adaptive state-feedback stabilization of high-order stochastic systems with nonlinear parameterization," Automatica, vol. 45, no. 1, pp. 126-133, 2009.

[30] X. Xie and N. Duan, "Output tracking of high-order stochastic nonlinear systems with application to benchmark mechanical system," IEEE Transactions on Automatic Control, vol. 55, no. 5, pp. 1197-1202, 2010.

[31] W. Li, X. Xie, and S. Zhang, "Output-feedback stabilization of stochastic high-order nonlinear systems under weaker conditions," SIAM Journal on Control and Optimization, vol. 49, no. 3, pp. 1262-1282, 2011.

[32] X. Xie, N. Duan, and X. Yu, "State-feedback control of highorder stochastic nonlinear systems with SiISS inverse dynamics," IEEE Transactions on Automatic Control, vol. 56, no. 8, pp. 1921-1926, 2011.

[33] W. Li, X. Liu, and S. Zhang, "Further results on adaptive state-feedback stabilization for stochastic high-order nonlinear systems," Automatica, vol. 48, no. 8, pp. 1667-1675, 2012.

[34] X. Xie and L. Liu, "A homogeneous domination approach to state feedback of stochastic high-order nonlinear systems with time-varying delay," IEEE Transactions on Automatic Control, vol. 58, no. 2, pp. 494-499, 2013.

[35] F. Li and Y. Liu, "Global stability and stabilization of more general stochastic nonlinear systems," Journal of Mathematical Analysis and Applications, vol. 413, no. 2, pp. 841-855, 2014.

[36] C. Zhao and X. Xie, "Global stabilization of stochastic highorder feedforward nonlinear systems with time-varying delay," Automatica, vol. 50, no. 1, pp. 203-210, 2014. 


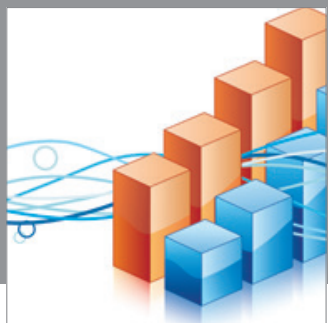

Advances in

Operations Research

mansans

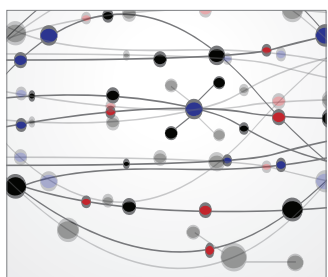

The Scientific World Journal
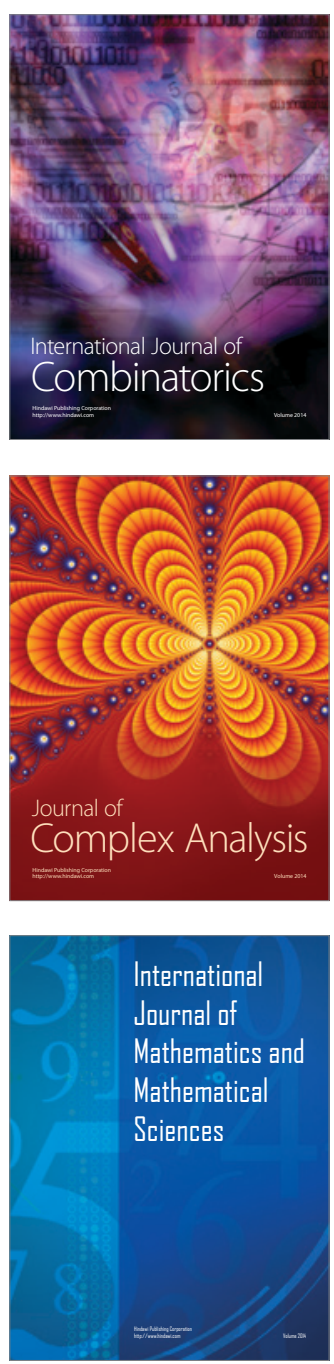
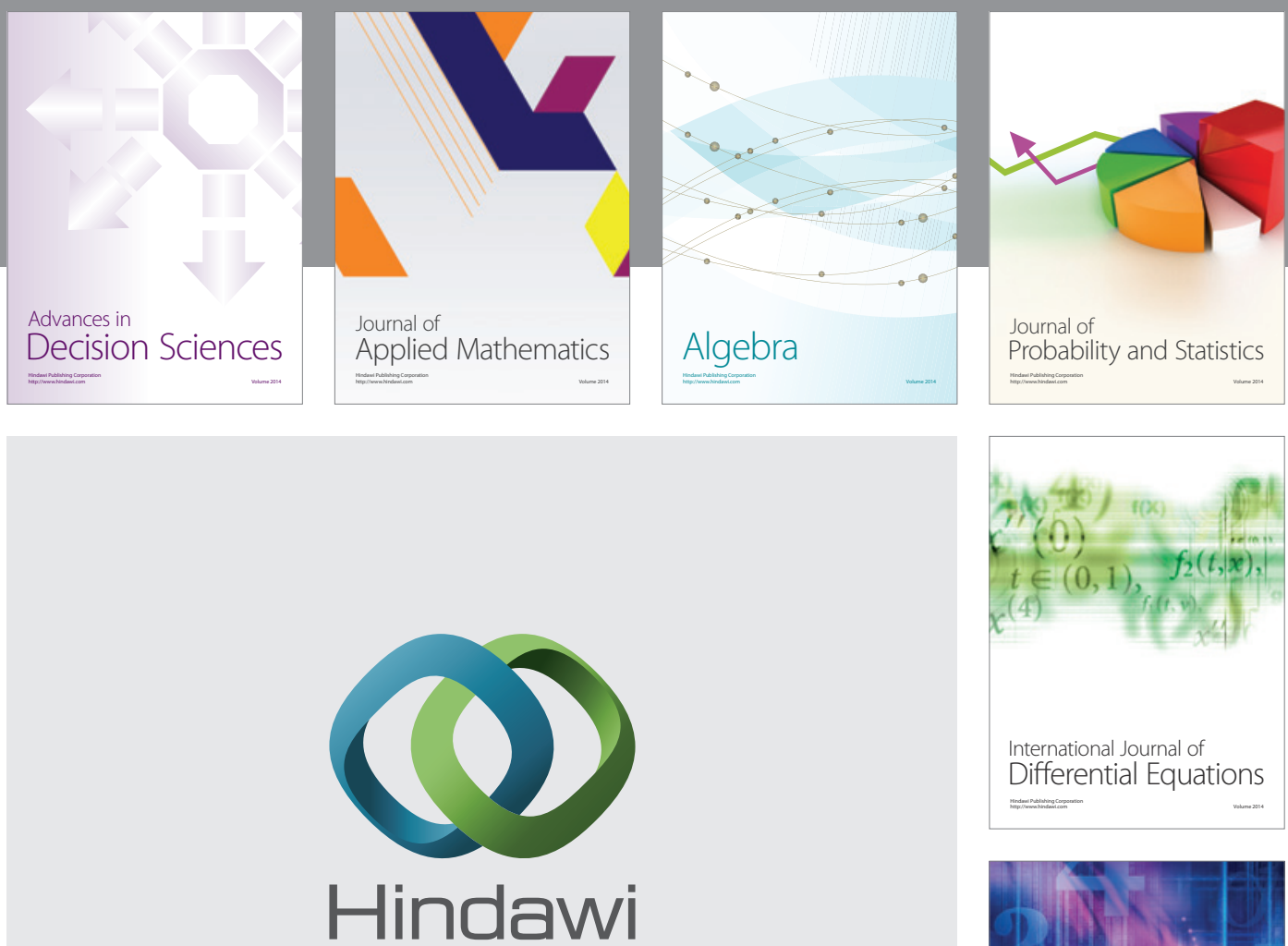

Submit your manuscripts at http://www.hindawi.com
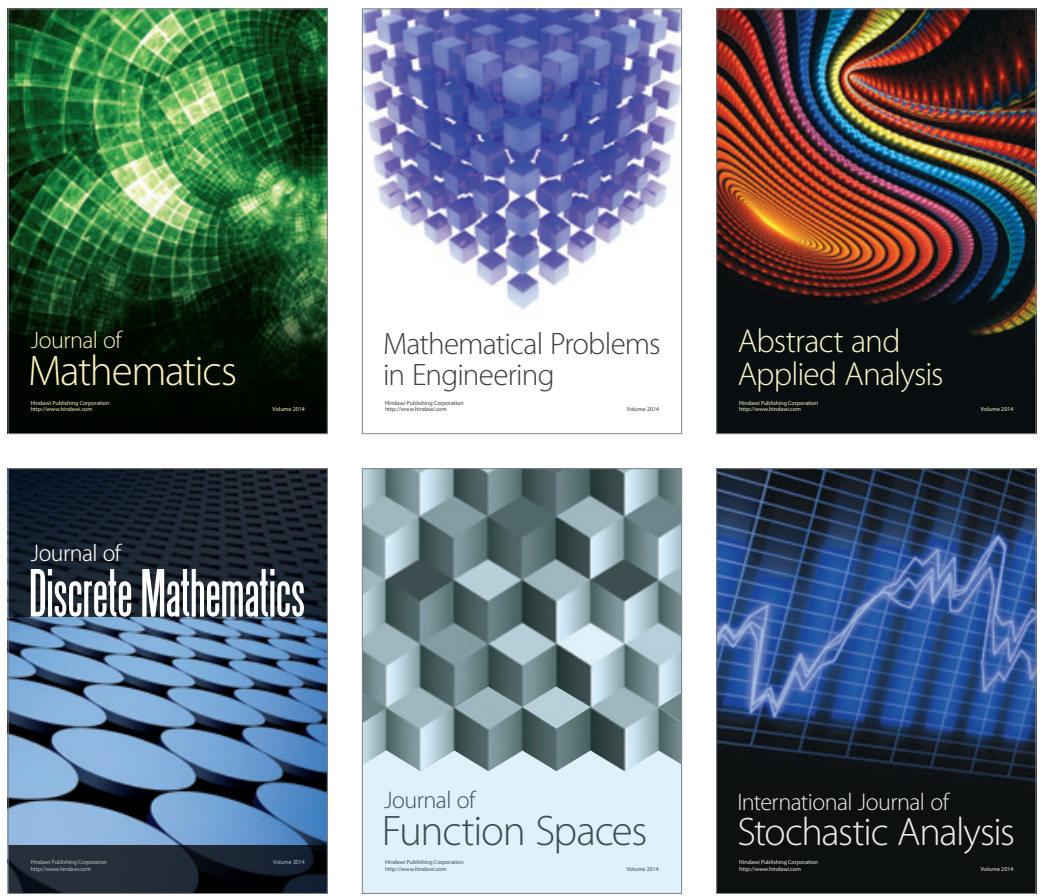

Journal of

Function Spaces

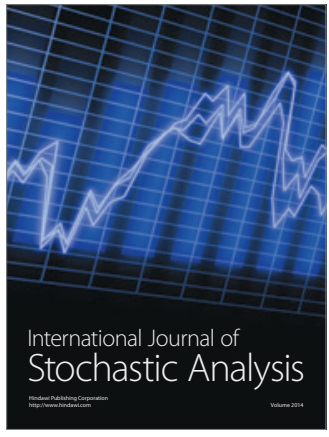

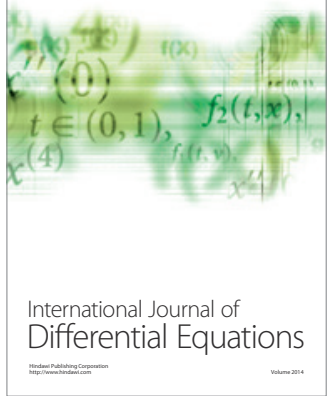
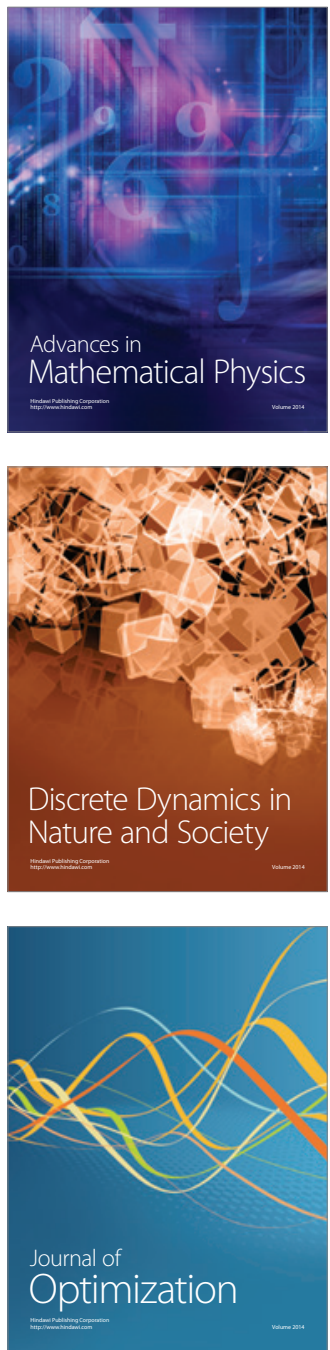